\title{
Research and Prospect on Healthy Meat Consumption of Urban and Rural Residents
}

\author{
Xiao Ying*, Liu Senhui, Cao Jianmin \\ College of Economic Management, Jilin Agricultural University, Changchun, China \\ Email address: \\ 532912404@qq.com (Xiao Ying) \\ ${ }^{*}$ Corresponding author
}

To cite this article:

Xiao Ying, Liu Senhui, Cao Jianmin. Research and Prospect on Healthy Meat Consumption of Urban and Rural Residents. European Business \& Management. Vol. 4, No. 3, 2018, pp. 88-91. doi: 10.11648/j.ebm.20180403.14

Received: July 25, 2018; Accepted: August 23, 2018; Published: September 25, 2018

\begin{abstract}
Meat consumption has always been an important issue concerned by socio-economic researchers. Meat is recognized by most consumers for its ability to provide nutrients other than grains. As the economy improves, residents choose to consume more meat products. Of course, some consumers think that too much meat consumption is bad for their health, they will choose to reduce their consumption of meat products. Combined with the influence of regional culture and national customs, meat consumption varies greatly from country to country and even from country to country. So, is there a healthy standard for meat consumption? This issue has attracted the attention of all countries in the world and reached a consensus conclusion: it is suggested that the consumption structure of cereals and meat should be balanced and reasonable. Most countries have developed scientific dietary guidelines based on the health of their citizens. However, examine the dietary guidelines standards and the actual meat consumption of residents in various countries, it can be found that some residents have a serious shortage of meat consumption, and the meat consumption of other residents far exceeds the guideline recommendation. Many scientific studies have found that under consumption of meat and overconsumption of meat can cause a variety of diseases, the end result will not only consume the accumulation of personal wealth but also hinder the overall development of the country. So, how to guide the healthy development of meat consumption should be a special concern of governments and researchers. Given the importance of healthy meat consumption, this study reviews the influencing factors of meat consumption in recent years and the guiding policies of healthy meat consumption in various countries, this paper studies the healthy meat consumption of urban and rural residents in China, and puts forward the research plan and plan for the future research of this problem.
\end{abstract}

Keywords: Urban and Rural Residents, Meat Consumption, Health

\section{Evolution of World Meat Consumption and Regional Consumption Characteristics}

Global meat consumption is on an increasing trend, but different levels of meat consumption growth are different. In general, total meat consumption in developing countries as a proportion of total world consumption is gradually increasing, for the first time, meat consumption in developing countries exceeded that of developed countries in 1994, and then it goes straight up. At the beginning of the 21 st century, meat consumption in developing countries reached 139.3 million tons, accounting for 56 percent of total world consumption. Meat consumption in developed countries increased to 104.6 million tons in 1990, and has not increased in a straight line since then. In terms of meat consumption per capita, regional differences are relatively obvious. Asia is a region with relatively low per capita meat consumption but rapid growth. For example, the per capita meat consumption in Taiwan in China increased rapidly from $16.2 \mathrm{~kg}$ in 1960 to $79 \mathrm{~kg}$ in 2000 . Japan increased from $6.5 \mathrm{~kg}$ in 1960 to $43.5 \mathrm{~kg}$ (excluding consumption of aquatic products) in 2009, an increase of 5.69 times. But USDA statistics show, the average per capita meat consumption in Taiwan from 2013 to 2015 was $72.2 \mathrm{~kg}$, while the average per capita meat consumption in Japan from 2013 to 2015 was $47.5 \mathrm{~kg}$. Obviously, meat consumption in these 
two regions has not increased rapidly in recent years, and some regions even show a decreasing trend. Europe has a high level of meat consumption per capita. The average meat consumption in countries like Canada and the United States is more than $90 \mathrm{~kg}$. USDA statistics show, from 2013 to 2015, the average U.S. meat consumption per capita was $111.1 \mathrm{~kg}$, and the growth rate was almost zero, Canada's meat consumption per person has fallen to $81.3 \mathrm{~kg}$ in the past three years. It can be seen that countries (regions) with excessive meat consumption have begun to gradually reduce meat consumption.

\section{Actual Meat Consumption and Healthy Meat Consumption of Urban and Rural Residents in China}

Our country has the largest population in the world, the first dietary guidelines were proposed by the Chinese nutrition society in 1989 . In 2014, according to the needs of residents' nutrition development, Formulated the outline of China's food and nutrition development (2014-2020), emphasizes that residents should consume $29 \mathrm{~kg}$ of meat and its products every year (including pork, beef, mutton, poultry, etc. except aquatic products). According to this standard and the actual meat consumption of urban and rural residents in China, the industrial economy research office analyzes the healthy meat consumption of urban and rural residents in China. The laboratory attempts to use "healthy meat consumption $(\mathrm{H})=$ actual meat consumption $/ 29$ " as a measure of healthy meat consumption of residents, the closer the index is to 1 , the healthier meat consumption is, and the greater the deviation from 1, the weaker the health consumption is.

\subsection{Healthy Meat Consumption of Urban Residents in China}

Urban residents have convenient conditions for meat consumption, when the income level is gradually improved, the total meat consumption is gradually increased, but the health meat consumption condition first improves and then deteriorates with the improvement of economic conditions. China's urban residents consumed $25.2 \mathrm{~kg}$ of meat per person in 1990, the $H$ value is 0.87 , the standards recommended in the dietary guidelines have not been met; By about 2005, The per capita meat consumption of urban residents reached $32.9 \mathrm{~kg}$, the $\mathrm{H}$ value was 1.13 , and the meat consumption exceeded the health standard. Since 2005 , the consumption of meat by urban residents in China has been increasing, healthy meat consumption is deteriorating. In 2015 , the number of meat per capita in urban households reached $38.3 \mathrm{~kg}$, which exceeded $32 \%$ of the healthy meat consumption standard. It's worth noting that, these are just meat consumption data for residential households, When considering the outdoor consumption of urban residents (Chen Qiong's research found that urban residents spend more than 40 percent of their outdoor meat consumption), the meat consumption of urban residents has far exceeded the health standards recommended by the dietary guidelines [1].

Of course, the above data show the average meat consumption of urban residents, even though the average level has far exceeded the health standard, there are significant differences in meat consumption among urban residents of different income groups. In 2010, meat consumption in the low-income and lower-middle income groups of urban residents was still insufficient, and there was still a gap between healthy consumption standards. Meat consumption in households of middle income group, upper middle income group and high income group reached over $28 \mathrm{~kg}$, these three groups of meat consumption and health consumption standards are similar. In 2011, meat consumption in all five income groups reached over $28 \mathrm{~kg}$, meat consumption in the lower income and lower middle income groups is close to health consumption standards, but the middle income level, upper middle income level and high income level residents meat consumption is moving away from health standards. Similarly, meat consumption increased in all five income groups in 2012, the higher the income, the greater the amount of meat consumed, and the greater the deviation from health standards.

\subsection{The Actual Consumption of Meat and Health of Rural Residents}

Compared with the meat consumption of urban residents, the per capita meat consumption of rural residential households is relatively low, but it also shows a rising trend. In 1990, the per capita meat consumption in rural residential households was only $12.6 \mathrm{~kg}$, until about 2005, it exceeded 20 $\mathrm{kg}$, it reached an all-time high in 2015, the average per capita meat consumption was $30.2 \mathrm{~kg}$. So, according to the standards recommended in the dietary guidelines, rural residents have a lower level of healthy meat consumption, it was not until 2015 that the per capita healthy meat consumption $(\mathrm{H})$ of rural residents reached the standard, generally speaking, the meat consumption of rural residents is characterized by insufficient consumption.

In terms of income groups, the characteristics of insufficient meat consumption of rural residents are more obvious. In 2010 from low income to high income group meat consumption levels did not reach health standards, the $\mathrm{H}$ value of healthy meat consumption in the high-income group was 0.87 , while the $H$ value of healthy meat consumption in the low-income group was only 0.56 , this suggests that the meat consumption of low-income rural people is only slightly more than half of the healthy level. 2011 and 2012 were similar, only the high-income groups consumed $27.7 \mathrm{~kg}$ of meat and $28.1 \mathrm{~kg}$ of meat respectively, getting closer to health standards. Obviously, the healthy consumption of meat by rural residents is different from that of urban residents. Increasing meat consumption is the core of improving the healthy consumption of meat by rural residents. 


\section{Research on Healthy Meat Consumption and the Guidance of Countries on Healthy Meat Consumption}

\subsection{Major Research on Healthy Meat Consumption}

Meat consumption is a free choice behavior of micro individuals, understanding the influencing factors of meat consumption is the key to guide meat health consumption scientifically. Income and price are the basic factors influencing healthy meat consumption, the understanding of the relationship between income and meat consumption has been reached as follows: in a particular period of time or a shorter economic cycle, it can be seen as a qualitative variable with specific development rules and supports the view that income levels are significantly correlated with meat consumption. Liu [2] used China's rural and urban household survey data to conduct an empirical study on meat consumption in Chinese households through AIDS model, found that increased income not only changes the amount of consumption but also changes the composition of meat consumption. Economic theory says, the relationship between price and meat consumption is relatively simple, there is a negative correlation between meat product price and consumption, there is a positive relationship between alternative prices and meat consumption, but the relationship between the price of complementary goods and meat consumption is negative. Some researchers [3-4] have also calculated the proportion of meat consumption through micro data, summarized the characteristics of animal products consumed by urban and rural residents, and evaluated the dietary nutrition of urban residents through the contribution of nutritional value of animal products. Foreign research focuses more on the interaction between price and consumption. Wang [5] used VAR model to study the endogeneity of demand and price of American meat (beef, poultry and pork), it is concluded that the demand consumption and price of beef and poultry meat influence each other, however, the relationship between the amount of pork consumed and the price: the price affects demand unilaterally.

In addition, some domestic researchers pay attention to the effects of meat product quality safety, freshness, brand, product label information, portability and other attributes on meat consumption, the results show that the characteristics of these products significantly influence consumers' willingness to pay and their choice behavior [1, 6-7]. In addition to focusing on economic conditions and the impact of meat product attributes on consumption, in view of the insufficient meat consumption of some residents, another part of the residents of the situation of excessive meat consumption, many researchers have explored the influence of urbanization, social structure change, consumer cognition change and other factors brought about by social development on the healthy consumption of meat [8-10]. Huang Jikun's research points out [11], changes in lifestyle and dietary preferences as a result of urbanization as well as changes in market development and occupation play a decisive role in changing the structure of China's food demand, and will continue to influence future changes in China's food consumption structure. Liu lei [12] directly introduced urbanization into the analysis model, it is found that urbanization has a stable positive stimulation effect on the growth of meat consumption, and this positive effect has a lag effect. With the further development of social economy, the cognitive level of reasonable diet will have a significant impact on the demand for meat [13], the research on the influence of consumer health concern and other factors on meat consumption has become one of the hot topics.

\subsection{Research on Intervention Policies of Meat Consumption in Developed Countries}

The main challenge for healthy meat consumption in developed countries is to guide excessive meat consumption. More and more national and regional governments are realizing, the quality of nutritional health directly affects the quality of the whole people and the economic development of the national society. After the Second World War, the world began to study and focus on residents of dietary nutritional problems, the United Nations food and agriculture organization (FAO) and the world health organization (WHO) as early as the $1970 \mathrm{~s}$ of main nutrients for residents in different countries put forward the reasonable composition proportion. The United States has the highest per capita meat consumption in the world, making it the world's highest caloric intake. In view of the domestic high levels of meat consumption, The United States established a nutrition testing system in 1977 to update dietary guidelines on a regular basis. The US government in 2011 introduced a complete heat and nutrition information, called "my plate" healthy dietary guidelines, residents can develop a nutritious and healthy personalized diet based on their weight and physical activity [14]. Britain also was once a typical high quantity of heat, high protein, high fat western dietary pattern, in recent 10 years the British government by making consumption, including "traffic light food labels" (Require distributors and food producers in the food label use like traffic light is red, yellow, green 3 kinds of color, representing this kind of food of fat, saturated fat, sugar and salt content high, medium and low three levels) guidance and intervention measures, gradually bring meat intake close to recommended levels [15].

\section{Research Prospect}

Obviously, from the perspective of revision of national dietary guidelines and national dietary intervention policies with high meat consumption per capita, the healthy consumption of meat will be the scientific dietary choice of future residents, but how to guide and stimulate the healthy meat consumption of the residents, both to avoid insufficient consumption and to prevent excessive consumption, this is a complex system project. Previous studies have used mature econometric research methods to conduct in-depth and 
systematic research on consumers' meat payment intention and meat selection behavior, the research results are of great reference value. However, meat consumption and health consumption (consume according to health standards) are fundamentally different, past research results in guiding residents health meat consumption may not meet expectations, and even bring the opposite result. In view of the meat consumption quantity and product attribute, economic factors and there are complex relations between factors such as social development, especially a lot of meat consumption of urban and rural residents in China do not meet the condition of healthy consumption, rural residents mainly lack of meat consumption while urban residents are the problem of excessive meat consumption. It is necessary to separate the two groups of insufficient meat consumption and excessive meat consumption, establish the indicators of healthy meat consumption, long term tracking of the consumption behavior of two types of consumers, it establish panel data and analyzes that health consume behavior of meat and its determining factors through the metering model system. In particular, we draw on the experience of the us and the UK, we will study scientific and operable intervention policies so as to guide the meat consumption behavior of urban and rural residents more effectively.

\section{References}

[1] Chen Qiong. study on meat consumption of urban and rural residents [D]. Beijing: Chinese Academy of Agricultural Sciences, 2010.

[2] Liu H, Parton K A, Zhou Z Y, et al. At-home meat consumption in China: an empirical study [J]. Aust J Agr Resour Ec, 2009, 53:485-501.

[3] Yang zhenni, Zhou Lin, Llu Shijun, Cheng Guangyan. Characteristics of consumption of animal products and dietary nutrition of urban residents [J]. China animal husbandry magazine, 2016, 52 (22):9-15.

[4] Cheng Guangyan, Liu Shanshan, Yang Zhenni, Wang Dongyang. Characteristics of China's meat consumption and 2020 forecast analysis [J]. China's rural economy, 2015 (02):76-82.
[5] Wang Z J, Besslerb DA. Price and quantity endogeneity in demand analysis evidence from directed acyclic graphs $[\mathrm{J}]$. Agrc Econ, 2006, 34: 87-95.

[6] Zou Yingheng, Wang Xiaoqing, Geng Hui. consumer analysis of the purchase behavior of added information traceable beef based on the survey of the Carrefour supermarket in Shanghai [J]. China rural economy, 2008, (5): 22-32.

[7] Wang Wenzhi, Wu La Ping. urban residents' willingness to pay for the quality and safety attributes of pork [J]. Agricultural technology economy, 2013, (11): 24-31.

[8] Ye Feng, Xie Juan, Ma Jjinggui. Analysis on the impact of urbanization on meat consumption of urban residents [J]. Journal of changjiang university (self-published), 2015, 15 (14):73-77.

[9] Chen Haiyan. Structural evolution and policy choice of China's meat processing industry $[\mathrm{J}]$. China journal of animal husbandry, 2008, 54 (03):114-119.

[10] Ding Cunzhen, Xiao Haifeng. A study on the characteristics of variables and their contribution to the decomposition of China's meat production [J]. World agriculture, 2017 (06):142-149.

[11] Huang Jikun. Social development, urbanization and food consumption [J]. Chinese Social Sciences, 1999, (4): 102-116.

[12] Liu Lei. research on meat consumption in China from the perspective of urbanization [D]. Beijing: Chinese Academy of Agricultural Sciences, 2014.

[13] Yen S T, Lin B H, Davis C G. Consumer knowledge and meat consumption at home and away from home [J]. Food Policy, 2008, 33:631 -639.

[14] Cheng Guangyan, Liu Shanshan. Analysis of the characteristics of food supply and demand in the United States and the dietary consumption of residents $[\mathrm{J}]$. world agriculture, 2013, (12):12-16.

[15] Yang Zhenni, Zhou Lin, Cheng Guang Yan. Characteristics of food consumption and dietary nutrition of British residents [J]. world agriculture, 2015, (1): 97-101. 